\title{
VACUNAS Y AUTOINMUNIDAD: UNA RARA ASOCIACIÓN BAJO DEBATE
}

\author{
Alexander Batista-Duharte ${ }^{1, a}$
}

\begin{abstract}
RESUMEN
La posible asociación entre vacunas y enfermedades autoinmunes es un tema controversial. Existen elementos a favor de esta relación basados en modelos teóricos, ensayos de laboratorio y varios casos clínicos publicados. En cambio, los estudios epidemiológicos no han confirmado esta asociación y, de ellos, puede inferirse que las vacunas no constituyen una causa demostrada de enfermedades autoinmunes. En este trabajo se analizan las evidencias a favor y en contra de esta controversial asociación, además, se aborda un nuevo síndrome asociado con la administración continuada de adyuvantes vacunales. Se concluye que debido al gran impacto en beneficio de la salud logrado con las vacunas, es necesario continuar desarrollando esta tecnología, pero también se debe seguir perfeccionando los diseños de las nuevas formulaciones y profundizando estudios básicos, preclínicos, ensayos clínicos y farmacovigilancia de los nuevos candidatos vacunales para establecer el riesgo real de desarrollo de un evento autoinmune posvacunación.
\end{abstract}

Palabras clave: Vacunas; Adyuvantes inmunitarios; Enfermedad autoinmunes; Autoinmunidad (Fuente: DeCS BIREME).

\section{VACCINES AND AUTOIMMUNITY: A STRANGE ASSOCIATION UNDER DEBATE}

\begin{abstract}
The occurrence and significance of autoimmune manifestations after administration of vaccines remain controversial. Evidence for immunization triggered autoimmunity come from several sources including theoretical models, animal studies, single and multiple case reports. In contrast, several epidemiological studies don't report this association, which is reassuring and at least indicates that vaccines are not a major cause of autoimmune diseases. We analyzed current scientific data concluded that vaccines bring a positive impact on public health, so it is necessary to continue developing this technology. Evaluation methods should be improved to avoid or anticipate the possible autoimmune side effects that can be presented.
\end{abstract}

Key words: Vaccines; Adjuvants, immunologic; Autoimmune diseases; Autoimmunity (Source: MeSH NLM).

\section{INTRODUCCIÓN}

La demostrada asociación entre infección y autoinmunidad en individuos genéticamente predispuestos (1), ha abierto el debate en la comunidad científica acerca de la posibilidad de que las vacunas también puedan inducir fenómenos autoinmunes ${ }^{(2)}$. Existen numerosos informes de pacientes que han desarrollado enfermedades autoinmunes después de aplicarles determinadas vacunas, ello hace sospechar una relación causal, pero son varios los estudios epidemiológicos que no han confirmado esta asociación, lo cual ha generado un intenso debate sobre este tema ${ }^{(3)}$.

La posibilidad de que una vacuna sea capaz de inducir un evento autoinmune no debe soslayarse, aunque la magnitud del riesgo potencial es aparentemente baja. En la actualidad aún son limitados los conocimientos relacionados con la etiología, la base genética y otros factores de riesgo que forman parte del mosaico de las enfermedades autoinmunes, por lo que este constituye un campo de intensa investigación seguido de cerca por las entidades regulatorias y de salud en todos los países.

En este artículo se abordan las evidencias a favor y en contra, y se discuten los mecanismos inmunológicos que, sobre la base de los conocimientos actuales, pudieran estar participando en esta hipotética asociación.

\section{MODELOS EXPERIMENTALES DE INDUCCIÓN DE ENFERMEDAD AUTOINMUNE POR INMUNIZACIÓN}

Existen diversos modelos de enfermedades autoinmunes, inducidos en animales de laboratorio por inmunización con

\footnotetext{
Laboratorio de Inmunotoxicología, Centro de Toxicología y Biomedicina (TOXIMED), Universidad de Ciencias Médicas. Santiago de Cuba, Cuba.

a Médico inmunólogo doctor en Medicina
}

Recibido: 16-01-12 Aprobado: 21-03-12 
antígenos obtenidos de sus propios tejidos formulados con adyuvantes. Un ejemplo clásico es el modelo de encefalitis alérgica experimental (EAE), el cual se obtiene en líneas de ratas y ratones susceptibles, al inocular extractos de tejido del sistema nervioso central, proteína básica de mielina (PBM) o péptidos derivados de ella con adyuvante completo de Freund (ACF), con lo que se logra un cuadro neurológico característico ${ }^{(4)}$. Otros modelos se han descrito para reproducir una enfermedad autoinmune en animales de laboratorio, siguiendo protocolos similares, es decir, inoculando autoantígenos del tejido contra el cual queremos obtener la respuesta autoinmune, formulados con un inmunoadyuvante ${ }^{(5)}$. Sin embargo, se ha descrito que la inoculación de un péptido procedente de la polimerasa del virus de la hepatitis $B$, que comparte similitudes secuenciales con la PBM de conejos, al ser inoculado a estos produjo infiltración perivascular localizada en el sistema nervioso central, semejante a la que ocurre con la inoculación de la PBM completa o péptidos encefalitogénicos derivados de esta, demostrándose, además, una respuesta inmune humoral y celular contra la propia PBM ${ }^{6}$.

Los modelos experimentales ofrecen una base para estudiar los mecanismos de un posible desarrollo de enfermedad autoinmune posvacunal. Sin embargo, hay que considerar que dichos modelos no reproducen exactamente lo que sucede cuando se aplica una vacuna en humanos, ya que se basan en esquemas de inmunización en condiciones extremas, empleando adyuvantes como los de Freund, no aprobados para uso clínico; además, se utilizan casi siempre modelos genéticamente susceptibles y se inmuniza con homogenizados de órganos o autoantígenos purificados, lo cual no ocurre en la práctica.

\section{SOSPECHAS DE INDUCCIÓN DE AUTOINMUNIDAD POR VACUNAS HUMANAS Y ESTUDIOS EPIDEMIOLÓGICOS}

Son numerosos los casos sospechosos de enfermedades autoinmunes posvacunación documentados en la literatura médica. De igual modo, existen bases de datos y publicaciones periódicas que registran los eventos adversos asociados a vacunas como: US Vaccine Adverse Events Reporting System (VAERS), y REACTIONS respectivamente, que permiten procesar y analizar estadísticamente la relación causal entre las vacunas y sus efectos adversos, incluyendo los eventos autoinmunes posvacunación (7).

\section{VACUNAS CONTRA HEPATITIS}

El primer caso de sospecha de asociación de vacunas antihepatitis B con signos de desmielinización del sistema nervioso central en humanos se registró en $1991^{(8)}$. Luego, en Francia, se describieron 35 casos de enfermedades desmielinizantes que ocurrieron en un período de ocho semanas después de aplicada una vacuna similar, confirmándose después que en la mitad de los casos se trataba de esclerosis múltiple ${ }^{(9)}$. La acumulación de casos de esclerosis múltiple espontánea llevó al ministro francés de salud pública a prohibir temporalmente el programa de vacunación de escolares con la vacuna de hepatitis B iniciado en 1995, lo que unido a la ausencia de datos epidemiológicos que demostraran lo contrario, creó mucho debate y confusión ${ }^{(10)}$. Posteriormente se realizaron estudios epidemiológicos y otros análisis que no pudieron confirmar esta relación causal ${ }^{(11-12)}$.

Girard ha cuestionado los métodos que se emplearon y las conclusiones emitidas luego de los estudios controlados, señalando que no se recogieron todos los casos de autoinmunidad asociada a esta vacuna y lo inadecuado de los métodos estadísticos usados (13). Por otro lado, Bordanos et al. informaron que varios pacientes vacunados contra hepatitis $B$ desarrollaron anticuerpos contra péptidos miméticos de la proteína oligodendrocítica de mielina; sin embargo, no se detectaron consecuencias clínicas de esta reactividad cruzada y los anticuerpos disminuyeron su título en los meses posteriores. En este mismo estudio no se detectó anticuerpos contra péptidos de la PBM ${ }^{(14)}$. Esto apoya el criterio de que no siempre una reacción autoinmune tiene una traducción clínica, lo cual complica más la interpretación de los resultados. Aún se debate sobre el peligro real de la vacunación contra hepatitis $B$ y en general se acepta que de existir en la práctica una verdadera relación causal, los beneficios que se informan por esta vacuna superan el posible riesgo.

Se conoce la existencia de fenómenos autoinmunes asociados con la infección natural por el virus de la hepatitis A pero en relación con las vacunas contra hepatitis $A$, hasta la fecha, se ha informado un caso de una posible conexión con la hepatitis autoinmune ${ }^{(15)}$, por lo que esta vacuna es considerada una de las más seguras, luego de millones de dosis aplicadas en todo el mundo.

\section{VACUNA CONTRA INFLUENZA}

El síndrome de Guillain Barré (SGB) también denominado poliradiculoneuritis desmielinizante, está estrechamente relacionado con una infección previa con agentes como Camphylobacter jejuni, virus de Epstein-Barr (VEB), virus de la hepatitis $B$ y cytomegalovirus, con evidencias de reacción inmune cruzada entre antígenos virales y antígenos mielínicos y constituye la afección autoinmune más frecuente a la que se le ha adjudicado una relación con vacunas antiinfluenza ${ }^{(16)}$. 
La relación del SGB con la vacuna $A / N e w$ Jersey de influenza, se sugirió luego de una campaña de vacunación realizada en los Estados Unidos entre 1976 y 1977, durante la cual se incrementó el número de casos de esta afección en las primeras semanas después de la aplicación de la vacuna, particularmente en la quinta semana, disminuyendo la incidencia luego de la sexta, lo que conllevó a una suspensión temporal del programa de vacunación (17). Por otro lado, una investigación retrospectiva desarrollada luego de campañas efectuadas en 1992-1993 y 1993-1994 reveló que la incidencia dentro de las seis semanas fue significativa aunque mucho más baja que en la campaña de los años $70{ }^{(18)}$. En otro estudio donde se utilizaron los datos de la VAERS, se encontró un incremento significativo del riesgo de padecer SGB luego de la vacunación contra influenza, existiendo diferencias entre los productores de la vacuna y las cepas virales empleadas ${ }^{(19)}$. En tanto, se ha demostrado que el riesgo de recurrencia de SGB luego de un episodio inicial y de reinmunización con vacuna de influenza, es mínimo.

\section{VACUNA CONTRA HEMÓPHILUS INFLUENZAE TIPO B}

Se ha sugerido que la inmunización con vacuna contra $H$. influenzae tipo b (Hib) después de los dos meses de edad, puede estar relacionada con la diabetes tipo 1 en humanos y que el riesgo potencial de la vacuna puede exceder el beneficio que brinda ${ }^{(20)}$. Sin embargo, en estudios controlados realizados no se ha confirmado esta asociación (21).

\section{VACUNA CONTRA FIEBRE AMARILLA}

La reemergencia de la fiebre amarilla ha conllevado a la vacunación masiva en países como Brasil, Australia, España, entre otros, con vacunas atenuadas como 17D y 17DD, las cuales han sido muy exitosas en su capacidad

Tabla 1. Informes seleccionados de asociaciones sospechosas o confirmadas entre vacunas y manifestaciones autoinmunes ${ }^{(7-24)}$

\begin{tabular}{|c|c|}
\hline Vacuna & Manifestaciones autoinmunes asociadas \\
\hline Hepatitis B & $\begin{array}{l}\text { Trastornos reumáticos: artritis reumatoidea, artritis reactiva, exacerbación de LES, síndrome de Sjógren, } \\
\text { vasculitis, crioglobulinemia, poliartralgia, poliarteritis nudosa, mialgia, fatiga. } \\
\text { Reacciones cutáneas: eritema nudoso, eritema multiforme, lupus eritematoso cutáneo, esclerodermia localizada, } \\
\text { liquen plano. } \\
\text { Trastornos neurológicos: encefalitis, meningitis aséptica aguda, mielitis transversa, neuritis óptica, neuropatía } \\
\text { de los plexos lumbar y braquial, síndrome de Guillain-Barre, parálisis de Bell, ataxia cerebelar aguda, miastenia } \\
\text { grave, esclerosis múltiple. } \\
\text { Trastornos hematológicos: trombocitopenia, anemia hemolítica immune, anemia aplásica } \\
\text { Otras manifestaciones: síndrome nefrótico, uveítis, alopecia, pericarditis aguda, enfermedad de Graves. }\end{array}$ \\
\hline Hepatitis A & Hepatitis autoinmune \\
\hline Influenza & $\begin{array}{l}\text { Trastornos reumáticos: purpura de Henoch-Schoenlein, poliangitis microscópica, artritis reactiva, vasculitis de } \\
\text { células gigantes, polimialgia reumática, crioglobulinemia, poliartralgia, fatiga. } \\
\text { Trastornos neurológicos: meningoencefalitis/encefalitis, neuritis óptica, mielitis transversa, neuritis braquial, } \\
\text { síndrome de Guillain-Barre, parálisis de Bell, } \\
\text { Trastornos hematológicos: linfocitopenia, trombocitopenia, anemia hemolítica autoimmune, } \\
\text { Trastornos cardíacos: miocarditis aguda, pericardítis aguda. } \\
\text { Otras manifestaciones: diabetes mellitus tipo I, síndrome nefrótico, uveítis, miositis, eritema multiforme. }\end{array}$ \\
\hline $\begin{array}{l}\text { Parotiditis/ } \\
\text { Rubeola/ } \\
\text { Sarampión }\end{array}$ & $\begin{array}{l}\text { Trastornos reumáticos: artritis y artralgia aguda, artritis crónica, miositis. } \\
\text { Trastornos neurológicos: encefalitis, meningitis aséptica, mielitis, neuritis óptica, síndrome de Guillain-Barre. } \\
\text { Trastornos hematológicos: trombocitopenia/purpura trombocitopénica aguda, síndrome hemolítico urémico, } \\
\text { anemia hemolítica. }\end{array}$ \\
\hline Varicela & $\begin{array}{l}\text { Trastornos reumáticos: artritis o artralgia, vasculitis (usualmente Henoch-Schoenlein) } \\
\text { Trastornos neurológicos: encefalitis, meningitis aséptica, mielitis, neuritis óptica, síndrome de Guillain-Barre, } \\
\text { ataxia cerebelar transitoria, neuritis óptica, mielitis transversa, síndrome de Guillain-Barre, parálisis de Bell. } \\
\text { Trastornos hematológicos: trombocitopenia, anemia aplásica. } \\
\text { Cutánea: eritema multiforme. }\end{array}$ \\
\hline $\begin{array}{l}\text { Vacuna } \\
\text { antipoliomielítica }\end{array}$ & $\begin{array}{l}\text { Trastornos reumáticos: artritis aguda. } \\
\text { Trastornos neurológicos: encefalitis, síndrome de Guillain-Barre. } \\
\text { Trastornos hematológicos: anemia hemolítica autoinmune. }\end{array}$ \\
\hline Rabia & $\begin{array}{l}\text { Trastornos neurológicos: Síndrome de Guillain-Barre, mielitis, meningitis, autoanticuerpos contra sustancia } \\
\text { blanca, proteína básica de mielina y cardiolipina en relación directa con las complicaciones neurológicas. } \\
\text { Otras: Cuadro similar a enfermedad del suero }\end{array}$ \\
\hline Viruela & $\begin{array}{l}\text { Trastornos neurológicos: encefalopatía posvacunal, encefalomielitis. } \\
\text { Otras: eritema multiforme, miopericarditis, cardiomiopatía dilatada. }\end{array}$ \\
\hline $\begin{array}{l}\text { Difteria/ toxoide } \\
\text { tetánico }\end{array}$ & $\begin{array}{l}\text { Trastornos neurológicos: enfermedades desmielinizantes del sistema nervioso central, síndrome de Guillain- } \\
\text { Barré. }\end{array}$ \\
\hline $\begin{array}{l}\text { Enfermedad de } \\
\text { Lyme }\end{array}$ & \\
\hline BCG & Artritis, enfermedad de Reiter. \\
\hline Fiebre amarilla & Enfermedad viscerotrópica y neurotrópica* (* mecanismo no precisado) \\
\hline
\end{tabular}


protectora; sin embargo, la aparición de casos muy esporádicos de cuadros denominados enfermedades viscerotrópicas y neurotrópicas, han propiciado algunos debates sobre su seguridad ${ }^{(22)}$. Aún no se ha determinado el mecanismo de estos efectos adversos severos producidos por estas vacunas ${ }^{(23)}$, aunque parece que las personas de más de 60 años, y aquellas con alguna predisposición o compromiso inmunológico exhiben un mayor riesgo de estos eventos adversos considerados raros, por lo que se propone evaluar en estas poblaciones el riesgo/beneficio de la vacunación ${ }^{(24)}$. Estas vacunas en general se consideran seguras y altamente efectivas contra esta peligrosa enfermedad.

Además de los casos anteriores existen otras sospechas de asociaciones entre vacunas específicas y procesos autoinmunes. La Tabla 1 resume los informes más debatidos.

\section{SÍNDROME AUTOINMUNE / INFLAMATORIO INDUCIDO POR ADYUVANTES}

Recientemente Shoenfeld y Agmon-Levin han propuesto un nuevo síndrome que se conoce con las siglas ASIA (autoimmune/inflammatory syndrome induced by adjuvants), que incluye cuatro entidades

Tabla 2. Criterios sugeridos para el diagnóstico del síndrome autoinmune / inflamatorio inducido por adyuvantes (ASIA)*

\section{CRITERIOS DIAGNÓSTICOS}

\section{Criterios mayores}

- Exposición a un estímulo externo (infección, vacuna, silicona, adyuvantes), previo a las manifestaciones clínicas.

- Manifestaciones clínicas típicas

- Mialgia, miositis o debilidad muscular;

Artralgia o artritis;

- Fatiga crónica con trastornos del sueño;

Manifestaciones neurológicas (especialmente asociadas a desmielinización):

- Trastornos cognitivos, pérdida de la memoria; Pirexia, sequedad de los labios.

Criterios menores

- Presencia de autoanticuerpos o anticuerpos contra el adyuvante sospechoso;

- Otras manifestaciones clínicas como el síndrome del colon irritable;

- HLA específicos en el paciente (Ej. HLA DRB1, HLA DQB1);

- Desarrollo de una enfermedad autoinmune (Ej. esclerosis múltiple).

* Esta es una propuesta de criterios diagnósticos de ASIA ${ }^{(25)}$ que aún debe ser validada.

(Traducido y reproducido con autorización de sus autores Shoenfeld y Agmon-Levin). denominadas: 1) siliconosis; 2) síndrome de la guerra del Golfo; 3) miofascitis macrofágica y 4) fenómeno posvacunación asociado a adyuvantes; las cuales comparten varios criterios clínicos y, además, tienen como denominador común la exposición previa a inmunoadyuvantes ${ }^{(25)}$. La Tabla 2 resume según sus autores, los criterios para el diagnóstico de este potencial síndrome.

Aún existen varios aspectos que deben ser esclarecidos para aceptar definitivamente esta propuesta como un nuevo síndrome. Los adyuvantes pueden aparentemente inducir un ASIA, sin embargo, todavía falta dilucidar los mecanismos celulares y moleculares que puedan explicar este fenómeno. De igual modo, la rareza de estos eventos sugiere la existencia de una susceptibilidad genética en las personas que la padecen, por lo que debe estudiarse qué genes pudieran estar implicados.

\section{VACUNACIÓN EN PACIENTES PORTADORES DE ENFERMEDADES AUTOINMUNES}

Una preocupación latente de las autoridades sanitarias y regulatorias es la posibilidad de que una vacuna agrave una enfermedad autoinmune preexistente. En este sentido, varios estudios controlados, particularmente en pacientes con esclerosis múltiple, artritis reumatoidea y LES, utilizando vacunas contra hepatitis $B$, influenza, neumococo y toxoide tetánico no han revelado evidencias de agravamiento de la enfermedad posvacunación ${ }^{(10)}$, por lo que, hasta el momento no existen criterios objetivos que permitan dictaminar que un paciente portador de una enfermedad autoinmune no deba ser vacunado.

\section{MECANISMOS QUE JUSTIFICAN UNA POSIBLE ASOCIACIÓN VACUNA-AUTOINMUNIDAD}

El desarrollo de un proceso autoinmune descansa en la influencia de factores genéticos asociados con factores externos que inducen o facilitan la pérdida de la inmunotolerancia con el desarrollo de respuestas inmunes contra estructuras propias. El factor genético determina que no todos los individuos tienen la misma predisposición a desarrollar una enfermedad autoinmune, entre los genes mayormente involucrados se encuentran los que codifican los HLA (del inglés: Human Leucocyte Antigens). Existen reportes de asociación de ciertos haplotipos HLA con enfermedades autoinmunes, siendo el HLA-B27 el haplotipo que mayor asociación presenta con enfermedades como la espondilitis anquilosante ${ }^{(26)}$. Otros genes recientemente asociados con enfermedades autoinmunes, son el gen 
AIRE (del inglés: autoimmune regulator), que participa en la presentación de autoantígenos durante el proceso de tolerancia central en el timo, para la deleción de linfocitos $T$ autorreactivos, cuyo defecto puede llevar al desarrollo de enfermedades autoinmunes multisistémicas (27). También se han encontrado asociaciones con polimorfismos en el gen que codifica el CTLA-4 (del inglés: cytolytic T lymphocyteassociated antigen), un regulador negativo en la activación de linfocitos $\mathrm{T}$, y en la familia de proteínas TIM (del inglés: $T$ cell immunoglobulin and mucindomain-containing) ${ }^{(28)}$.

El mimetismo molecular en antígenos microbianos, pueden constituir un peligro potencial para el desarrollo de autoinmunidad posvacunal ${ }^{(1,6)}$, especialmente en presencia de un adyuvante vacunal. Los adyuvantes optimizan la respuesta inmune contra el antígeno coadministrado, pero también contribuyen a la toxicidad de las vacunas ${ }^{(29)}$, por lo que existe la hipótesis de que ciertos clones de linfocitos autoreactivos, que en condiciones fisiológicas no reconoce antígenos propios por estar bajo la presión de la tolerancia periférica, bien pudiera incrementar su capacidad de reconocimiento y activación por la influencia de la actividad del adyuvante y desarrollar una respuesta autorreactiva, especialmente en individuos con un fondo genético favorable ${ }^{(30)}$ (Figura 1). Afortunadamente, los mecanismos de tolerancia son muy efectivos para limitar el efecto nocivo que pudiera causar el mimetismo molecular entre microorganismos y estructuras propias.

Otro mecanismo que puede actuar en la autoinmunidad posvacunal es la formación de complejos inmunes, causando vasculitis o la exacerbación de procesos autoinmunes latentes (10). Finalmente, hay que tener en cuenta que en la dinámica de la respuesta inmune normalmente se desarrollan respuestas contra estructuras propias de forma subclínica cuyo papel etiopatogénico aún se desconoce.

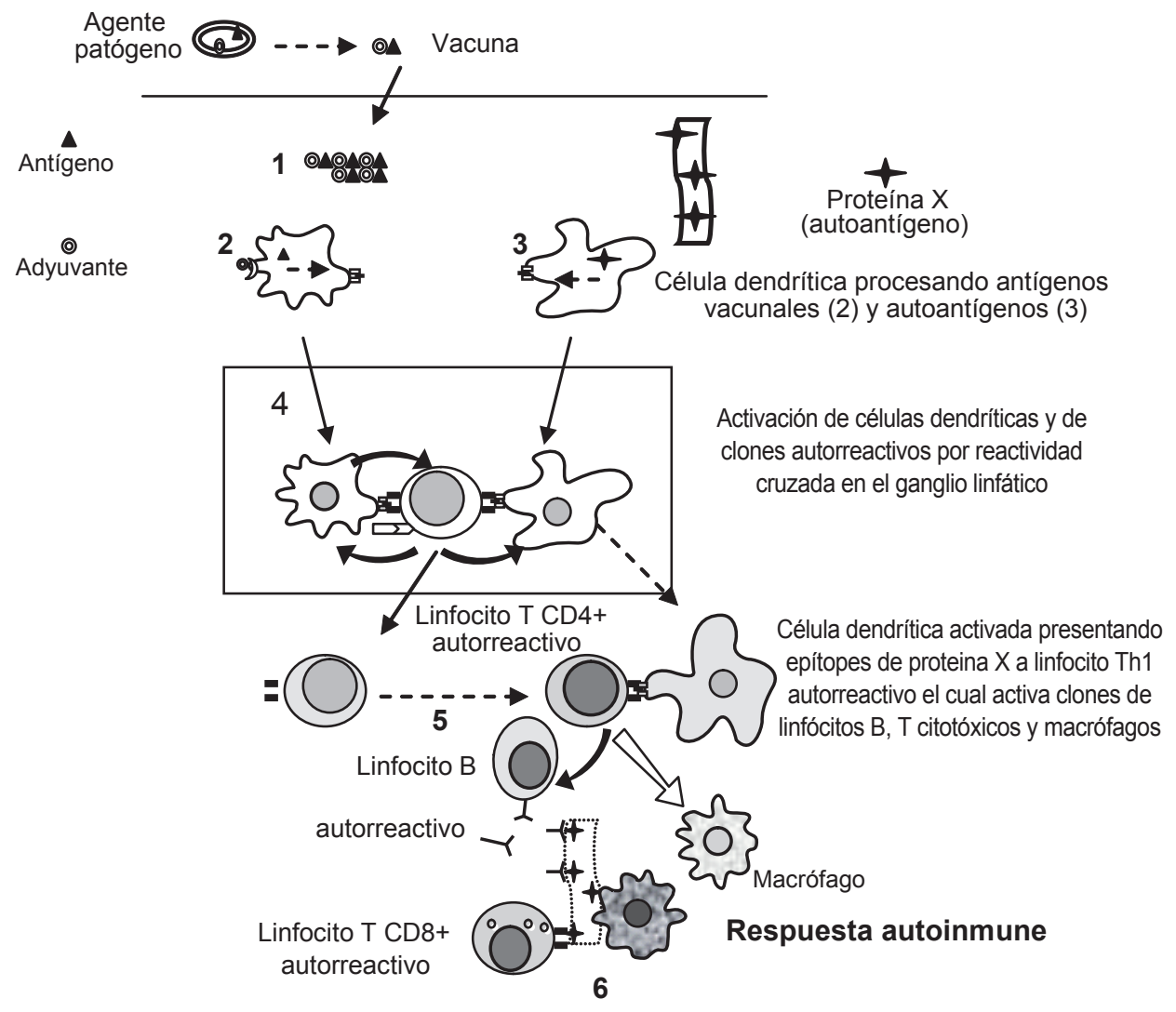

Figura 1. Mecanismo simplificado de posible ruptura de tolerancia en el entorno de una respuesta inmune vacunal.

(1) Antígenos vacunales con mimetismo molecular frente a estructuras propias, (1 y 2) presencia en la formulación de un adyuvante capaz de estimular en las células dendríticas (CD) los receptores de la inmunidad innata TLR (Toll like receptor) y NLRs (NOD-like receptors) que detectan PAMP (Pathogen-Associated Molecular Patterns) y otras "señales de daño" (3) CD procesando antígenos propios miméticos al antígeno vacunal. (4) Ambas $\mathrm{CD}$ presentan antígenos a clones autorreactivos los cuales son activados y estos activan a su vez a ambas CD. (5) Las CD que presentan antígenos propios ya activadas pueden continuar presentando y activando clones Th1 autorreactivos que, a su vez, pueden activar linfocitos B y T CD8+ también autorreactivos, así como macrófagos capaces de actuar sobre células propias dañadas, generándose un daño tisular autoinmune (6). 


\section{CONSIDERACIONES FINALES}

Aunque teóricamente, existe la posibilidad de inducción de una enfermedad autoinmune posvacunación y de la existencia de sospechas documentadas de asociaciones entre determinadas vacunas y afecciones autoinmunes, los estudios epidemiológicos realizados, en su mayoría, han concluido que no existe tal asociación o es de muy baja incidencia.

No obstante, las investigaciones epidemiológicas que se requieren en vacunas presentan hoy algunos problemas, como el desconocimiento del perfil genético de cada individuo vacunado, ya que todavía no han sido definidos con exactitud todos los genes implicados en la autoinmunidad. Esto hace suponer que probablemente el escaso porcentaje de casos que pudieran haber desarrollado una enfermedad autoinmune posvacunación, presentan una susceptibilidad genética que, de conocerse, podría en un futuro, cuando tecnológicamente sea posible, valorarse el riesgo-beneficio de determinadas vacunas en estas personas.

Otra dificultad en la interpretación de los estudios poblacionales es que resulta impredecible el momento en que se va a desarrollar una respuesta autoinmune, lo que unido a la imposibilidad de controlar los factores concurrentes, aun con el rigor de los estudios controlados y que algunos de ellos han tratado de extender las observaciones por varios años, queda la pregunta si ha sido suficiente el tiempo de observación. A esto podemos añadirle que hay que establecer vías para mejorar los registros de eventos adversos en los estudios de farmacovigilancia, ya que estos se realizan a través de una vigilancia pasiva en la cual el paciente espontáneamente es quien describe el evento, lo cual lleva implícito el problema de que muchas personas no realizan el informe $\mathrm{y}$, además, a medida que pasa el tiempo después de la vacunación es más difícil poder establecer una posible relación causal con un evento que aparece más tardíamente. Todo esto supone que es necesario perfeccionar los métodos de análisis y tener nuevos elementos en la patogenia de las enfermedades autoinmunitarias para poder establecer las estimaciones correctas.

\section{CONCLUSIÓN}

Debido al gran beneficio para la humanidad alcanzado con las vacunas, no hay justificación para detener o disminuir esta práctica. Sin embargo, el riesgo potencial de autoinmunidad posvacunal no puede ser ignorado $y$, aunque en la práctica no ha podido establecerse una clara relación causal, por las implicaciones éticas y médico-legales, constituye una responsabilidad de la ciencia seguir perfeccionando los métodos de evaluación desde el nivel básico hasta los estudios de farmacovigilancia y tratar de evitar al máximo los posibles efectos adversos, con un mayor rigor en el diseño y evaluación de los candidatos vacunales en desarrollo.

\section{Fuentes de financiamiento}

No se recibió financiamiento para la elaboración de este trabajo.

\section{Conflictos de interés}

El autor declara que no existen conflictos de interés.

\section{REFERENCIAS BIBLIOGRÁFICAS}

1. Rose NR. Autoimmunity, infections and adjuvants. Lupus. 2010;19(4):354-8.

2. Orbach H, Shoenfeld $\mathbf{Y}$. Vaccination, infection and autoimmunity: myth and reality VIAMR 2005-10-2628, Beau-Rivage Palace Hotel, Lausanne, Switzerland. Autoimmunity Rev. 2007(6):261-6.

3. Chen RT, Pless R, DeStefano F. Epidemiology of autoimmune reactions induced by vaccination. JAutoimmun. 2001;16(3):309-18.

4. Alvord EC, Kies MW, Suckling AJ. Experimental allergic encephalomyelitis: a useful model for multiple sclerosis. In : Alvord EC, Kies MW, Suckling AJ (Eds). Progress in Clinical and Biological Research. New York; Alan R Liss Inc; 1984. p. 523-37.

5. Burkhardt $\mathbf{H}$, Kalden JR. Animal models of autoimmune diseases. Rheumatol Int. 1997;17(3):91-9.

6. Oldstone MB. Molecular mimicry and immune-mediated diseases. FASEB J. 1998;12(13):1255-65.

7. Geier DA, Geier MR. Hepatitis B vaccination and arthritic adverse reactions: a follow-up analysis of the Vaccine Adverse Events Reporting System (VAERS) database. Clin Exp Rheumatol. 2002;20(1):119.

8. Herroelen L, de Keyser J, Ebinger G. Central-nervoussystem demyelination after immunisation with recombinant hepatitis B vaccine. Lancet. 1991;338(8776):1174-5.

9. Gout $O$, Lyon-Caen $O$. Sclerotic plaques and vaccination against hepatitis B. Rev Neurol (Paris). 1998;154(3):205-7.

10. Vial T, Descotes J. Autoimmune diseases and vaccinations. Eur J Dermatol. 2004;14(2):86-90.

11. Duclos P. Safety of immunization and adverse events following vaccination against hepatitis B. Expert Opin Drug Saf. 2003;2(3):225-31.

12. Schattner A. Consequence or coincidence? The occurrence, pathogenesis and significance of autoimmune manifestations after viral vaccines. Vaccine. 2005;23(30):3876-86.

13. Girard M. Autoimmune hazards of hepatitis B vaccine. Autoimmun Rev. 2005;4(2):96-100.

14. Bogdanos DP, Smith H, Ma Y, Baum H, Mieli-Vergani G, Vergani D. A study of molecular mimicry and immunological 
cross-reactivity between hepatitis B surface antigen and myelin mimics. Clin Dev Immunol. 2005;12(3):217-24.

15. Vento S, Garofano T, Di Perri G, Dolci L, Concia E, Bassetti D. Identification of hepatitis A virus as a trigger for autoimmune chronic hepatitis type 1 in susceptible individuals. Lancet. 1991;337(8751):1183-7.

16. Haber P, DeStefano F, Angulo FJ, Iskander J, Shadomy SV, Weintraub E, et al. Guillain-Barré syndrome following influenza vaccination. JAMA. 2004;292(20):2478-81.

17. Schoenberger LB, Bregman DJ, Sullivan-Bolyai JZ, Keenlyside RA, Ziegler DW, Retailliau HF, et al. GuillainBarre syndrome following vaccination in the National Influenza Immunization Program, United States, 1976-1977. Am J Epidemiol. 1979;110(2):105-23.

18. Lasky T, Terracciano Gj, Madger L, Koski CL, Ballesteros M, Nash D, et al. The Guillain-Barré syndrome and the 1992-1993 and 1993-1994 influenza vaccines. N Engl J Med. 1998;339(25):1797-802.

19. Geier MR, Geier DA, Zahalsky AC. Influenza vaccination and Guillain Barre syndrome small star, filled. Clin Immunol. 2003;107(2):116-21.

20. Childhood immunizations and type 1 diabetes: summary of an Institute for Vaccine Safety Workshop. The Institute for Vaccine Safety Diabetes Workshop Panel. Pediatr Infect Dis J. 1999;18(3):217-22.

21. Karvonen M, Cepaitis Z, Tuomilehto J. Association between type 1 diabetes and Haemophilus influenzae type $b$ vaccination: birth cohort study. BMJ. 1999;318(7192):116972.

22. Thomas RE, Lorenzetti DL, Spragins W, Jackson D, Williamson T. Active and passive surveillance of yellow fever vaccine 17D or 17DD-associated serious adverse events: systematic review. Vaccine. 2011;29(28):4544-55.

23. Barrett AD, Teuwen DE. Yellow fever vaccine - how does it work and why do rare cases of serious adverse events take place? Curr Opin Immunol. 2009;21(3):308-13.
24. Khromava AY, Eidex RB, Weld LH, Kohl KS, Bradshaw RD, Chen RT, et al. Yellow fever vaccine: an updated assessment of advanced age as a risk factor for serious adverse events. Vaccine. 2005;23(25):3256-63.

25. Shoenfeld Y, Agmon-Levin N. 'ASIA' - autoimmune/ inflammatory syndrome induced by adjuvants. J Autoimmun. 2011;36(1):4-8.

26. Braun J, Bollow M, Remlinger G, Eggens U, Rudwaleit M, Distler A, et al. Prevalence of spondyloarthropathies in HLA-B27 positive and negative blood donors. Arthritis Rheum. 1998; 41(1):58-67.

27. Pitkanen J, Peterson P. Autoimmune regulator: from loss of function to autoimmunity. Genes Immun. 2003;4(1):1221.

28. Anderson AC, Anderson DE. TIM-3 in autoimmunity. Curr Opin Immunol. 2006;18(6):665-9.

29. Batista-Duharte A, Lindblad EB, Oviedo-Orta E. Progress in understanding adjuvant immunotoxicity mechanisms. Toxicol Lett. 2011;203(2):97-105.

30. Waisbren BA Sr. Acquired autoimmunity after viral vaccination is caused by molecular mimicry and antigen complimentarity in the presence of an immunologic adjuvant and specific HLA patterns. Med Hypotheses. 2008;70(2):346-8.

Correspondencia: Alexander Batista-Duharte

Dirección: Autopista Nacional Km 1 11/2 Santiago de Cuba 4033, Cuba.

Teléfono: (53) 22643926

Correo electrónico: alexandro.batista@medired.scu.sld.cu

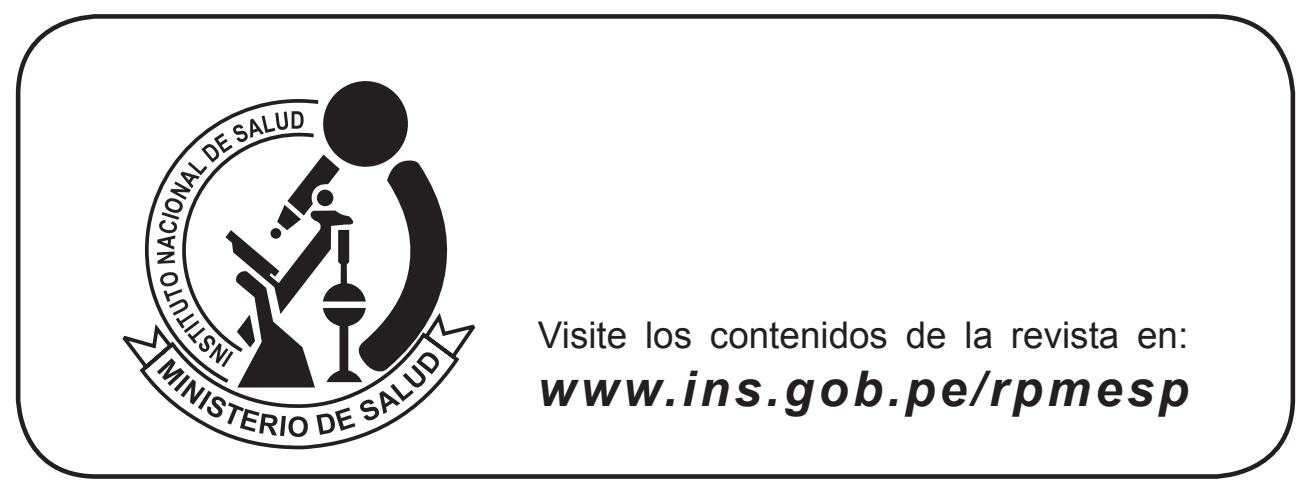

